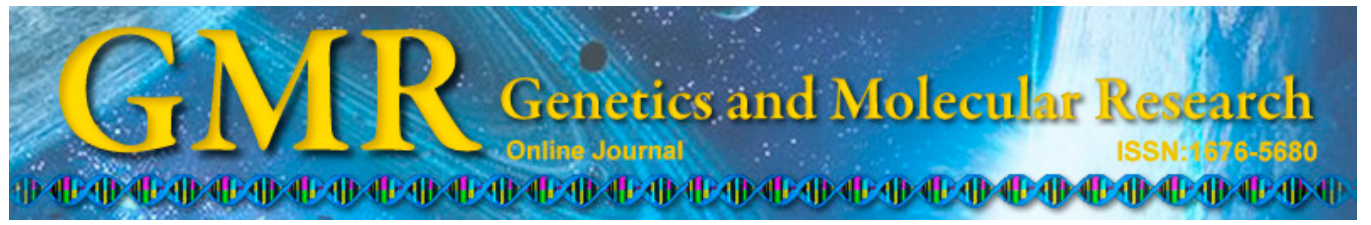

\title{
Effect of high-intensity exercise on interleukin-15 expression in rabbit synovia
}

\author{
Y.H. Wang, X.D. Li, W.B. Zhu and G.F. Sun \\ Department of Anesthesiology, Binzhou People's Hospital, Binzhou, \\ Shandong, China \\ Corresponding author: Y.H. Wang \\ E-mail: YuhaiWang6608@163.com
}

Genet. Mol. Res. 14 (4): 13852-13859 (2015)

Received January 26, 2015

Accepted June 8, 2015

Published October 29, 2015

DOI http://dx.doi.org/10.4238/2015.October.29.5

\begin{abstract}
The objective of this study was to examine the effect of high-intensity exercise on interleukin-15 (IL-15) expression in rabbit synovia. We utilized 24 New Zealand white rabbits, which were randomly divided equally into high-intensity exercise and control groups. The former were forced to run for $60 \mathrm{~min} /$ day over 4 weeks at the speed of 30 $\mathrm{m} / \mathrm{min}$. The histological changes of cartilage and knee joint synovia were investigated with hematoxylin and eosin staining. Immunohistochemistry and enzyme-linked immunosorbent assays were performed to measure IL-15 expression. From these analyses, we identified knee articular cartilage damage and synovitis in the high-intensity exercise group. This group also exhibited higher IL-15 expression in their synovial fluid and tissues than was observed in the control group $(\mathrm{P}<0.05)$. These results suggested that high-intensity exercise might lead to synovitis and articular cartilage damage, and that IL-15 overexpression in synovia might be associated with post-traumatic osteoarthritis.
\end{abstract}

Key words: Exercise; Interleukin-15; Osteoarthritis; Synovium; Synovial fluid 


\section{INTRODUCTION}

Inflammatory factors have a vital role in in the developmental process of traumatic arthritis (Wojdasiewicz et al., 2014). One such factor, interleukin 15 (IL-15), has received increasing amounts of attention. Originally described as a T cell growth factor, IL-15 is a kind of congenital immune response factor which modulates $\mathrm{T}$ - and natural killer cell activation and proliferation (Dubois et al., 2002). IL-15 plays important roles in mediating immune and inflammatory responses, and can regulate and mediate various pathophysiological processes in the body. Its roles in autoimmune disease and chronic inflammation in particular have recently been the focus of many studies. IL-15 can regulate osteoporosis (Neumann et al., 2005) and osteoclast formation during the process of bone destruction (Schett et al., 2005; Miranda-Carús et al., 2006; Pulsatelli et al., 2013). It can also modulate the functional maturation of dendritic cells, and can contribute to neutrophil and B cell activation and proliferation (Carroll et al., 2008; Moreno-Nieves et al., 2014). Previous studies have shown that IL-15 has important functions in various disorders such as rheumatic autoimmune disease (Pan et al., 2013), nervous inflammatory disease (Benito-Miguel et al., 2012), infection caused by total hip replacement (Saeed and Revell, 2001), and osteoarthritis (Maldonado and Nam, 2013). However, little research has been performed on the relationship between IL-15 and traumatic arthritis.

\section{MATERIAL AND METHODS}

\section{Animals}

Rabbits were used for all experiments, and all procedures were approved by the Animal Ethics Committee of Binzhou Municipal People's Hospital (Shandong, China).

In this study, we utilized 24 New Zealand white rabbits weighing $2.5-3.0 \mathrm{~kg}$. The rabbits were randomly divided equally into high-intensity exercise and control groups. Each rabbit was fed in a separate cage. The room temperature was maintained at $18^{\circ}-22^{\circ} \mathrm{C}$, and the humidity maintained at $50 \%$. None of the rabbits had previously undergone treadmill exercise testing.

The fitness exercise was conducted on a treadmill at a $30^{\circ}$ slope at $20 \mathrm{~m} / \mathrm{min}$ for 30 min, for 1 week. Canvas enclosure was used to prevent the escape of the animal. Following the fitness exercise, the 24 rabbits were randomly divided into normal activity (control) and high-intensity exercise groups, with 12 animals assigned to each group. The formal experiment began after 2 days of rest. The formal exercise was conducted at a $30^{\circ}$ slope. The initial speed was set to $10 \mathrm{~m} / \mathrm{min}$, gradually increased to $30 \mathrm{~m} / \mathrm{min}$ within $5 \mathrm{~min}$, and this speed was maintained for $60 \mathrm{~min}$. The exercise regime was applied once a day for 4 weeks. Rabbits in the high-intensity exercise group received mechanical drive or sound stimulation to make them continue moving during the experiment, while rabbits in the control group received unrestricted free activity.

\section{Sampling and index measurement}

Rabbits were anesthetized by administration of $5 \%$ ketamine and secured onto the operating table. A $1.0-\mathrm{mL}$ saline solution aliquot was injected into the right knee joint cavity. After repeated activity, $0.5 \mathrm{~mL}$ liquid was extracted from the knee joint and stored at $-80^{\circ} \mathrm{C}$. 
Enzyme-linked immunosorbent assay (ELISA) was used to determine the level of IL-15 expression in rabbit articular fluid according to the manual provided with the kit. Meanwhile, the femoral internal condyle and synovial tissue were also isolated from anesthetized rabbits and fixed in $10 \%$ formaldehyde solution, embedded in paraffin, sectioned at $5 \mathrm{~mm}$ and mounted on slides, and subjected to hematoxylin and eosin (HE) staining. The femoral internal condyle cartilage specimens were decalcified for 4 weeks, and then the HE staining was observed to identify potential synovial tissue and articular cartilage structure changes under a light microscope. The presence of IL-15 expression in rabbit synovia was determined by immunohistochemistry. The integral optical density (IOD) value of positively stained tissue was determined using an Olympus BX41 optical microscope (Tokyo, Japan) and the QImaging analysis system (QImaging Corporate, Surrey, BC, Canada). IL-15 immunohistochemical and ELISA kits were purchased from R\&D Systems (Minneapolis, MN, USA).

\section{Statistical analysis}

The SPSS 16.0 statistical software (SPSS, Chicago, IL, USA) was used for data analyses. Data are reported as means $\pm \mathrm{SD}$. The $t$-test was applied for independent samples, and statistically significant differences were defined as $\mathrm{P}<0.05$.

\section{RESULTS}

\section{General observations}

Upon microscopic examination, we found that the articular cartilage surfaces of the rabbits in the control group were smooth and silvery white with no obvious synovial hyperplasia. In contrast, the articular cartilage surfaces of the rabbits in the high-intensity exercise group appeared dim with local tiny cracks, while the synovia presented hyperemia, edema, and local adhesion.

\section{HE staining}

\section{Chondrocyte observation}

There were no obvious pathological changes in the articular cartilage surfaces and in the cartilage cells from the surface to the subchondral bone of the rabbits from the control group. The cell morphology changed gradually from spindle-shaped to circular. The cell arrangement changed from axial alignment to irregular (Figure 1A). In contrast, the articular cartilage surface in the rabbits from the high-intensity exercise group was observed to be uneven and with evident small cracks. The number of cartilage cells was reduced and these appeared clustered and exhibited stromal fibrosis (Figure 1B).

\section{Synovial tissue observation}

The synovial tissue cells showed regular arrangement in the rabbits in the control group, and could be divided into 1-2 layers. No inflammation or hyperplasia was observed (Figure 2A). On the other hand, the synovial tissue from the rabbits in the high-intensity ex- 
ercise group presented obvious hyperplasia, hyperemia, and edema. The synovial cells were disorderly and hyperplastic, and demonstrated infiltration by a large number of inflammatory cells (Figure 2B).

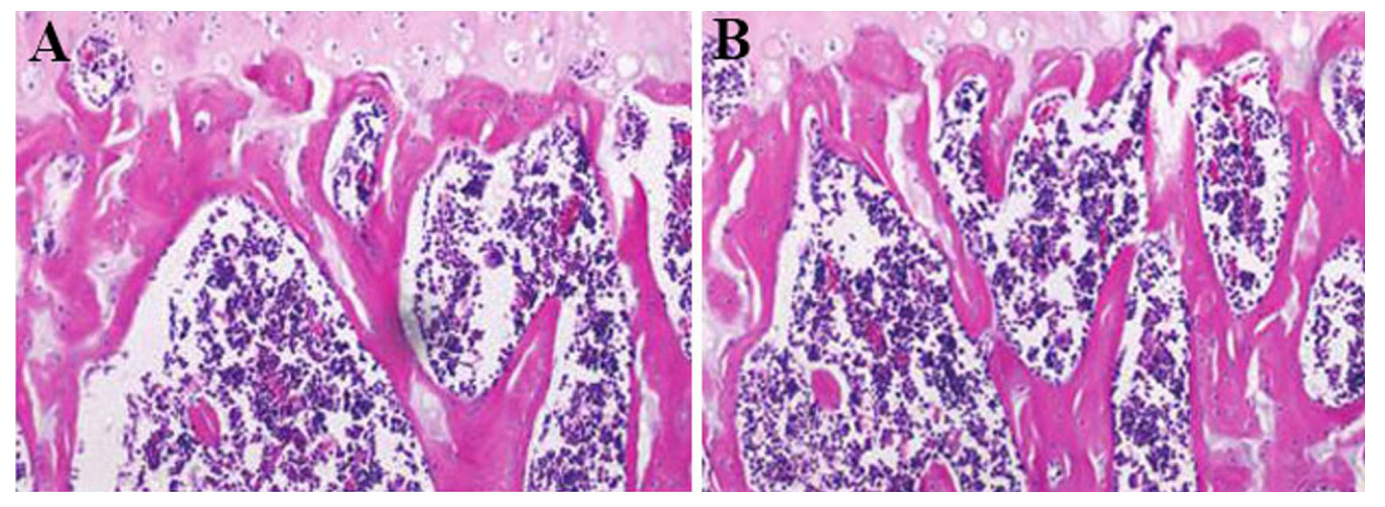

Figure 1. Chondrocytes in the two groups. A. Articular cartilage in the control group (HE staining, 400X). B. Articular cartilage in the high-intensity exercise group (HE staining, 400X). Dark purple-stained chondrocytes can be observed throughout the cartilage, primarily in subchondral bone. $\mathrm{HE}=$ hematoxylin and eosin.
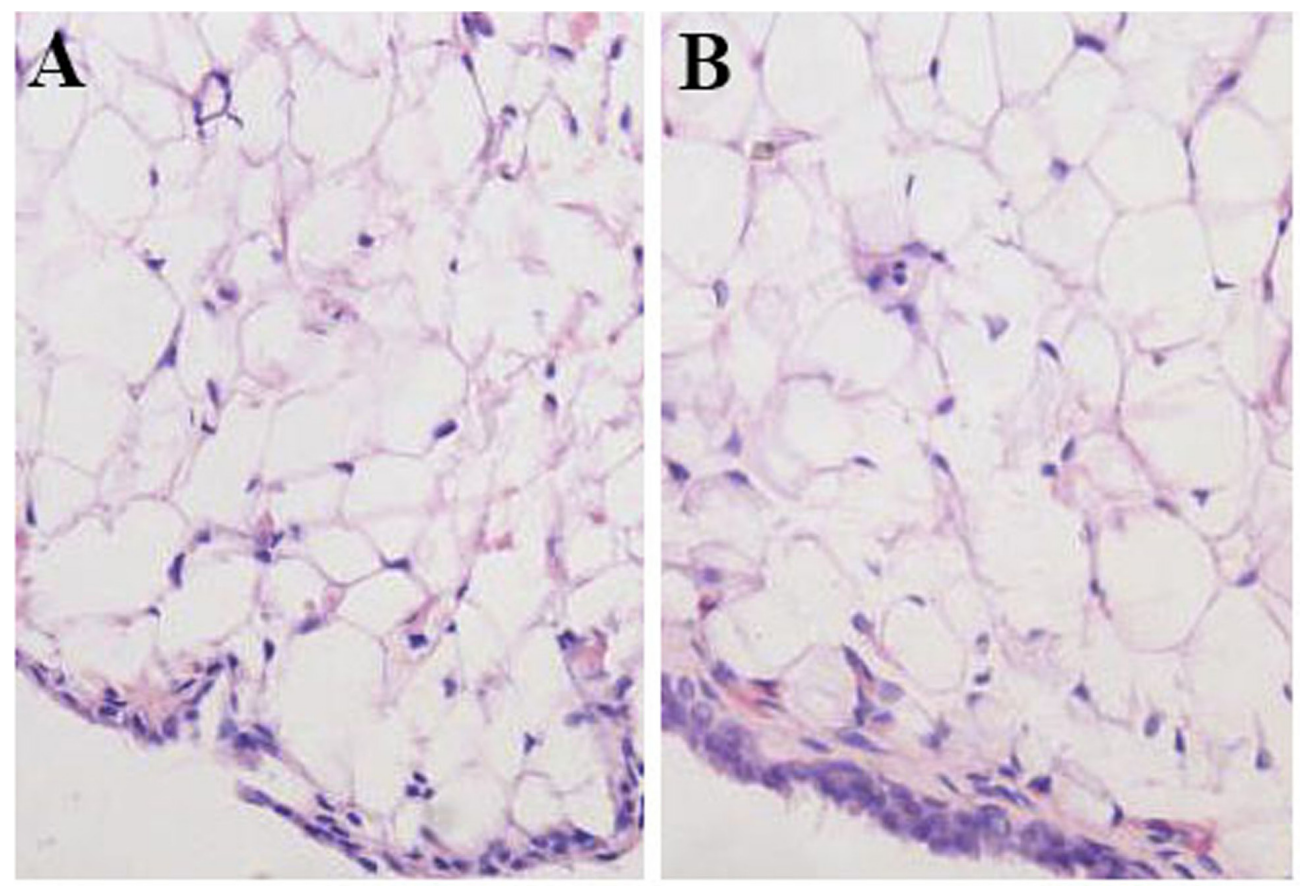

Figure 2. Synovial tissue in the two groups. A. Synovium of the joint in the control group (HE staining, 400X). B. Synovium of the joint in the high-intensity exercise group (HE staining, 400X). HE = hematoxylin and eosin. Nuclei and eosinophilic structures are stained by HE. Compared to control group, high-intensity exercise group presented obvious hyperplasia, hyperemia, and edema, as well as inflammatory cell infiltration. 


\section{IL-15 expression}

\section{IL-15-positive cell observation}

IL-15 showed only minimal expression in the rabbit synovia from the control group (Figure 3A). However, IL-15 was expressed at higher frequencies in the synovial cells, monocytes, lymphocytes, and vascular endothelial cells from the high-intensity exercise group as can be seen by the elevated numbers of cells exhibiting gray/brown-stained cytoplasm (Figure 3B). The IOD value of the synovial IL-15 was markedly higher in the high-intensity exercise group than that in the control group $(\mathrm{P}<0.01$; Table 1$)$. Coordinately, the IL-15 content in the synovial fluid increased significantly in the high-intensity exercise group as well $(\mathrm{P}<0.05$; Table 2$)$.

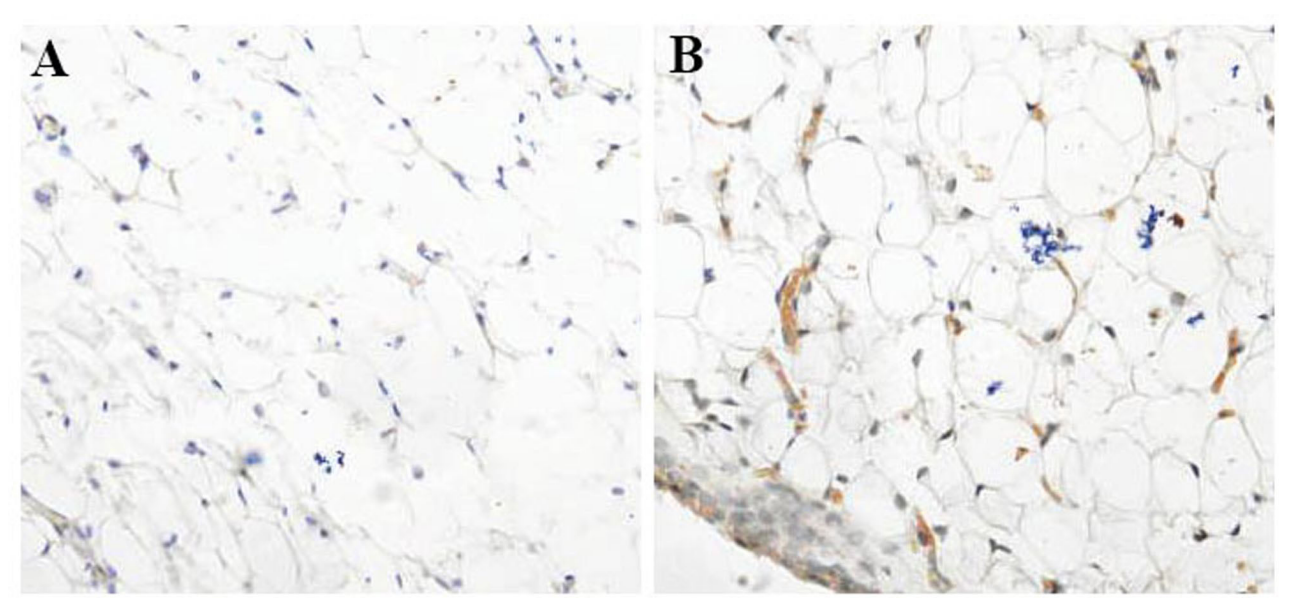

Figure 3. IL-15 expression in the two groups A. IL-15 expression in the synovium from the control group (immunohistochemical staining, 400X). B. IL-15 expression in the synovium from the high-intensity exercise group (immunohistochemical staining, 400X). Gray or brown indicates IL-15-positive signal. Blue indicates nucleus as stained by dopamine and (3,4-dihydroxyphenylamino)-2-imidazoline.

Table 1. IL-15-positive synovial cells in the two groups.

\begin{tabular}{lcr}
\hline Group & Subjects (N) & Synovial IOD \\
\hline Control group & 12 & $2.84 \pm 0.64$ \\
High-intensity exercise group & 12 & $13.22 \pm 0.82$ \\
$t$-test & & 8.95 \\
$\mathrm{P}$ & & $<0.05$ \\
\hline
\end{tabular}

IOD = integral optical density. Data are reported as means \pm standard deviation.

Table 2. IL-15 content in the synovial fluid of the two groups.

\begin{tabular}{lcr}
\hline Group & Subjects $(\mathrm{N})$ & $\mathrm{IL}-15 \mathrm{content}(\mathrm{pg} / \mathrm{mL})$ \\
\hline Control group & 12 & $40.18 \pm 3.27$ \\
High-intensity exercise group & 12 & $26.18 \pm 2.33$ \\
$t$-test & & 3.45 \\
P & & $<0.05$ \\
\hline
\end{tabular}

Data are reported as means \pm standard deviation. 


\section{DISCUSSION}

There are many situations that can lead to the occurrence of traumatic arthritis, and numerous studies have shown that the inflammatory cytokine-mediated inflammatory reaction is an important step in this process (Mazieres et al., 2006). IL-15 is an inflammatory cytokine of small molecular weight that primarily mediates inflammatory and immune responses, and promotes the expression of other inflammatory factors (Kinne et al., 2001). IL-15 is expressed in a variety of cell types including fibroblasts, keratinocyte, epithelial cells, osteoclasts, activated mononuclear cells, hematopoietic progenitor cells, marrow stromal cells, antigenpresenting cells, dendritic cells, and macrophages (Ruckert et al., 2003; Bulfone-Paus et al., 2006). IL-15 plays an important role in inflammatory and protective immune responses largely through regulation of cells in the immune system (Kapoor et al., 2011). IL-15 can induce B cell secretion of immunoglobulin ( $\operatorname{IgM}, \operatorname{IgG}$, and $\operatorname{IgA})$, and can promote $\mathrm{T}$ cell activation and chemotaxis, with concomitant production of cytokines and their receptors. IL-15 can promote $\mathrm{T}$ cell aggregation within the joint space and thus can aggravate the destruction of articular cartilage (Ruckert et al., 2009). Damaged joints leave the cartilage collagen bare, resulting in the induction of an autoimmune reaction and the further aggravation of cartilage damage (Goldring and Marcu, 2009). IL-15 can participate in this process through a variety of pathways, exacerbating the degeneration of the tissues and cartilage surrounded the joint. IL-15 can promote IL-1, IL-6, IL-17, and TNF- $\alpha$ expression, leading to the aggregation of numerous inflammatory factors in the region of inflammation, and the formation of a cascade amplification effect (Geng et al., 2008). IL-15 can also accelerate matrix metalloproteinase (MMP) secretion by cartilage cells, synovial cells, and fibroblasts. Proteolysis of the pro-peptide domain of MMP leads to its activation and diffusion to the cartilage, resulting in glycosaminoglycan loss of cartilage under stress load. This process eventually results in stress-related changes and articular cartilage degeneration and destruction (DiMicco et al., 2004). IL-15 is therefore of important significance in rheumatoid arthritis clinical research. IL-15 overexpression has been found both in joint tissue and synovial fluid (McInnes et al., 2003). Furthermore, inhibition of the excessive expression of IL-15 has been shown to prevent collagen-induced arthritis in a rodent model (Ruchatz et al., 1998).

Our experiment aimed to detect the IL-15 content in the synovial fluid of rabbits after high-intensity exercise, and to investigate its role in the process of traumatic arthritis. Our results showed that the knee joint synovial membrane and the articular cartilage suffered obvious injury including the infiltration of $\mathrm{T}$ lymphocytes and other inflammatory cells after high-intensity exercise. The articular cartilage surfaces of the rabbits from the high-intensity exercise group became uneven and presented small cracks. The cartilage cells were reduced in number, appeared to be clustered, and exhibited stromal fibrosis. Concomitantly, IL-15 was expressed in a larger number of synovial cells, monocytes, lymphocytes, and vascular endothelial cells from the high-intensity exercise group, as can be seen by the elevated numbers of cells exhibiting gray/brown-stained cytoplasm. Furthermore, the IL-15 content of the articular fluid increased significantly in the rabbits that had undergone high-intensity exercise than in the controls. In general, long-term high-intensity exercise usually results in articular cartilage injury. In our study, the exercise-associated injury, inflammation, and excessive expression of IL-15 appeared to be closely related and formed a chain reaction, which resulted in cartilage cell degeneration and eventually led to the occurrence of traumatic arthritis. 
IL-15 overexpression has been shown to be closely associated with the progression of traumatic arthritis. Its upregulation can aggravate joint destruction and synovial hyperplasia. Furthermore, previous studies have demonstrated that IL-15 expression is closely related to the degree of pain associated with arthritis (Sun et al., 2013). However, as single factors are not solely responsible for the occurrence of traumatic arthritis, it is necessary to further study the relationships between IL-15 and other inflammatory cytokines in future research.

\section{Conflicts of interest}

The authors declare no conflict of interest.

\section{ACKNOWLEDGMENTS}

We thank the anonymous reviewers for reviewing this manuscript.

\section{REFERENCES}

Benito-Miguel M, Garcia-Carmona Y, Balsa A, Bautista-Caro MB, et al. (2012). IL-15 expression on RA synovial fibroblasts promotes B cell survival. PloS One 7: e40620.

Bulfone-Paus S, Bulanova E, Budagian V and Paus R (2006). The interleukin-15/interleukin-15 receptor system as a model for juxtacrine and reverse signaling. Bioessays 28: 362-377.

Carroll HP, Paunovic V and Gadina M (2008). Signalling, inflammation and arthritis: Crossed signals: the role of interleukin-15 and -18 in autoimmunity. Rheumatology 47: 1269-1277.

DiMicco MA, Patwari P, Siparsky PN, Kumar S, et al. (2004). Mechanisms and kinetics of glycosaminoglycan release following in vitro cartilage injury. Arthritis Rheum. 50: 840-848.

Dubois S, Mariner J, Waldmann TA and Tagaya Y (2002). IL-15Ralpha recycles and presents IL-15 In trans to neighboring cells. Immunity 17: 537-547.

Geng H, Carlsen S, Nandakumar KS, Holmdahl R, et al. (2008). Cartilage oligomeric matrix protein deficiency promotes early onset and the chronic development of collagen-induced arthritis. Arthritis Res. Ther. 10: R134.

Goldring MB and Marcu KB (2009). Cartilage homeostasis in health and rheumatic diseases. Arthritis Res. Ther. 11: 224.

Kapoor M, Martel-Pelletier J, Lajeunesse D, Pelletier JP, et al. (2011). Role of proinflammatory cytokines in the pathophysiology of osteoarthritis. Nat. Rev. Rheumatol. 7: 33-42.

Kinne RW, Liehr T, Beensen V, Kunisch E, et al. (2001). Mosaic chromosomal aberrations in synovial fibroblasts of patients with rheumatoid arthritis, osteoarthritis, and other inflammatory joint diseases. Arthritis Res. 3: 319-330.

Maldonado M and Nam J (2013). The role of changes in extracellular matrix of cartilage in the presence of inflammation on the pathology of osteoarthritis. Biomed. Res. Int. 2013: 284873.

Mazieres B, Garnero P, Guéguen A, Abbal M, et al. (2006). Molecular markers of cartilage breakdown and synovitis at baseline as predictors of structural progression of hip osteoarthritis. The ECHODIAH Cohort. Ann. Rheum. Dis. 65: 354-359.

McInnes IB, Gracie JA, Harnett M, Harnett W, et al. (2003). New strategies to control inflammatory synovitis: interleukin 15 and beyond. Ann. Rheum. Dis. 62: ii51-4.

Miranda-Carús ME, Benito-Miguel M, Balsa A, Cobo-Ibáñez T, et al. (2006). Peripheral blood T lymphocytes from patients with early rheumatoid arthritis express RANKL and interleukin-15 on the cell surface and promote osteoclastogenesis in autologous monocytes. Arthritis Rheum. 54: 1151-1164.

Moreno-Nieves UY, Didier C, Lévy Y, Barré-Sinoussi F, et al. (2014). NK cells are primed by ANRS MVA(HIV)-infected DCs, via a mechanism involving NKG2D and membrane-bound IL-15, to control HIV-1 infection in CD4+ T cells. Eur. J. Immunol. 44: 2370-2379.

Neumann E, Gay S and Müller-Ladner U (2005). The RANK/RANKL/osteoprotegerin system in rheumatoid arthritis: new insights from animal models. Arthritis Rheum. 52: 2960-2967.

Pan W, Wu X, He Y, Hsuchou H, et al. (2013). Brain interleukin-15 in neuroinflammation and behavior. Neurosci. Biobehav. Rev. 37: 184-192. 
Pulsatelli L, Addimanda O, Brusi V, Pavloska B, et al. (2013). New findings in osteoarthritis pathogenesis: therapeutic implications. Ther. Adv. Chronic Dis. 4: 23-43.

Ruchatz H, Leung BP, Wei XQ, McInnes IB, et al. (1998). Soluble IL-15 receptor alpha-chain administration prevents murine collagen-induced arthritis: a role for IL-15 in development of antigen-induced immunopathology. J. Immunol. 160: 5654-5660.

Ruckert R, Brandt K, Bulanova E, Mirghomizadeh F, et al. (2003). Dendritic cell-derived IL-15 controls the induction of CD8 T cell immune responses. Eur. J. Immunol. 33: 3493-3503.

Ruckert R, Brandt K, Ernst M, Marienfeld K, et al. (2009). Interleukin-15 stimulates macrophages to activate CD4+ T cells: a role in the pathogenesis of rheumatoid arthritis? Immunology 126: 63-73.

Saeed S and Revell PA (2001). Production and distribution of interleukin 15 and its receptors (IL-15Ralpha and IL-R2beta) in the implant interface tissues obtained during revision of failed total joint replacement. Int. J. Exp. Pathol. 82: 201-209.

Schett G, Hayer S, Zwerina J, Redlich K, et al. (2005). Mechanisms of Disease: the link between RANKL and arthritic bone disease. Nat. Clin. Pract. Rheumatol. 1: 47-54.

Sun JM, Sun LZ, Liu J, Su BH, et al. (2013). Serum interleukin-15 levels are associated with severity of pain in patients with knee osteoarthritis. Dis. Markers 35: 203-206.

Wojdasiewicz P, Poniatowski LA and Szukiewicz D (2014). The role of inflammatory and anti-inflammatory cytokines in the pathogenesis of osteoarthritis. Mediators Inflamm. 2014: 561459. 